\title{
Cohesive Relations for Surface Atoms in the Iron-Technetium Binary System
}

\author{
Christopher D. Taylor \\ Materials Science and Technology Division, Los Alamos National Laboratory, Los Alamos, NM 87544, USA \\ Correspondence should be addressed to Christopher D. Taylor, cdtaylor@lanl.gov
}

Received 6 June 2011; Accepted 8 August 2011

Academic Editor: Livio Battezzati

Copyright (C 2011 Christopher D. Taylor. This is an open access article distributed under the Creative Commons Attribution License, which permits unrestricted use, distribution, and reproduction in any medium, provided the original work is properly cited.

Iron-technetium alloys are of relevance to the development of waste forms for disposition of radioactive technetium-99 obtained from spent nuclear fuel. Corrosion of candidate waste forms is a function of the local cohesive energy $\left(E_{\text {loc }}\right)$ of surface atoms. A theoretical model for calculating $E_{\text {loc }}$ is developed. Density functional theory was used to construct a modified embedded atom (MEAM) potential for iron-technetium. Materials properties determined for the iron-technetium system were in good agreement with the literature. To explore the relationship between local structure and corrosion, MEAM simulations were performed on representative iron-technetium alloys and intermetallics. Technetium-rich phases have lower $E_{\text {loc }}$, suggesting that these phases will be more noble than iron-rich ones. Quantitative estimates of $E_{\text {loc }}$ based on numbers of nearest neighbors alone can lead to errors up to $0.5 \mathrm{eV}$. Consequently, atomistic corrosion simulations for alloy systems should utilize physics-based models that consider not only neighbor counts, but also local compositions and atomic arrangements.

\section{Introduction}

The development of long-term containment strategies for spent nuclear fuel requires a combination of careful experiments and theoretical studies so that the realistic lifetimes of these strategies can be confidently predicted. Currently, a number of different containment strategies are being considered, one of which involves the storage of certain fission products within a metallic alloy waste form [1]. Chief among these fission products is technetium-99, which has a half-life of $\sim 10^{5}$ years [2]. Studies have demonstrated that technetium can be alloyed with stainless steel with significant mass-loadings and that these waste forms have considerable corrosion resistance [3].

Predictions of the long-term stability of this material can be obtained through the coupling of the results of accelerated testing studies with a rigorous, physics-based theoretical model. Corrosion is believed to be the chief process by which stored, spent nuclear fuel will degrade over time and have the potential to release radionuclides to the environment $[1,4,5]$. Modeling corrosion at long-time scales requires a thorough and fundamental understanding of the electrochemical stability of such systems, particularly the surface phenomena (Figure 1) [6]. Surface processes that are important to consider include passivation (formation of protective oxide films), depassivation (the rupture of such films by localized dissolution, mechanical damage, or presence of deleterious chemistries), mass-transport (at the metal/oxide interfaces, through the oxide, and at the oxide/solution interface), and bare-metal dissolution. The construction of a comprehensive corrosion model requires the elucidation of numerous processes acting across multiple length-scales and materials types (metals, oxides, and fluids).

One of the tools that can be applied to assist in the construction of lifetime prediction models is atomistic simulation. Contemporary atomistic modeling tools in the solid-state and molecular sciences allow the first-principles calculation of thermodynamic energies, activation barriers associated with phase-transformations, and the prediction of phase diagrams. First-principles modeling has been used by theorists at UNLV (Weck et al.) to explore the stability of Tc nanoparticles [7], and the nature of $\mathrm{Tc}-\mathrm{Zr}$ alloy materials 


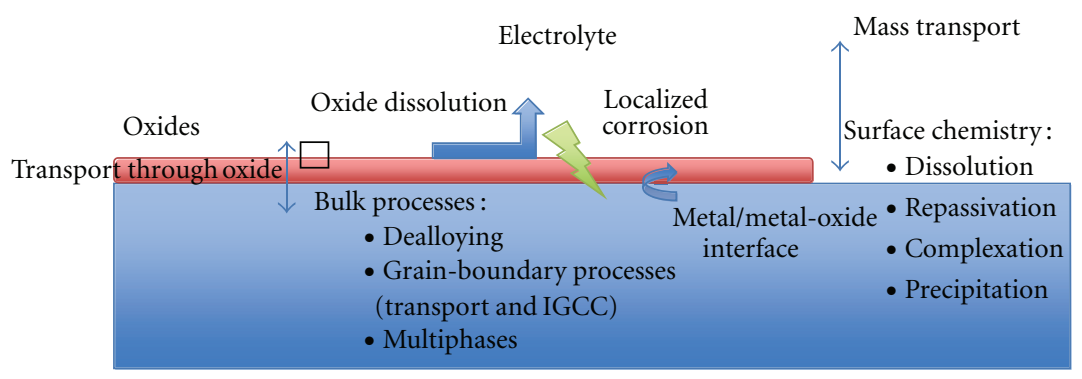

FIGURE 1: A schematic illustrating the many processes that need to be considered when building a model for the corrosion of candidate metal alloy waste forms.

[8]. First-principles models have also been used to address problems in Tc corrosion. In a previous study, for example, we considered the reaction of water with the metallic surfaces containing iron and technetium [9]. The reaction of water to form oxygen monolayers on metal surfaces is one of the first steps in the formation of metal oxide layers. Using density functional theory, we were able to compute the energy associated with the reaction of water with the surface to form hydroxide or oxide monolayers, and using this energy, we computed the surface coverage as a function of the electrochemical $\mathrm{pH}$ and potential.

In the present work, we have applied atomistic modeling to illuminate a second set of corrosion reactions relevant to the iron-technetium system. The tendency for an atom to dissolve or be released from the metal by other means (such as diffusion through the passive film or ligand complexation) is related to the intrinsic stability of the atom in its local surface configuration (composition, coordination number, and local orientation, see Figure 2). Metal atom surface stability is a fundamental property of metal atoms at the surface and can be quantified by the atomic cohesive energy. The cohesive energy of an atom can be determined using an embedding functional approach, such as the modified embedded atom method developed by Baskes and Johnson [10]. By developing a modified embedded atom potential for the iron-technetium binary system, we have then computed cohesive energies for surface metal atoms in this system and, subsequently, identified which atoms and configurations of atoms will be more prone to release from the system due to corrosion effects. We plan to use this tool in future simulations of the active dissolution of candidate irontechnetium alloy waste forms.

\section{Computational Details}

Our strategy for computing the dissolution tendency of metal atoms in the iron-technetium binary system required the calculation of cohesive energies using the modified embedded atom method. It was necessary for us to construct a modified embedded atom potential that is accurate for the iron-technetium interactions. Modified embedded atom potentials for binary systems can be constructed by

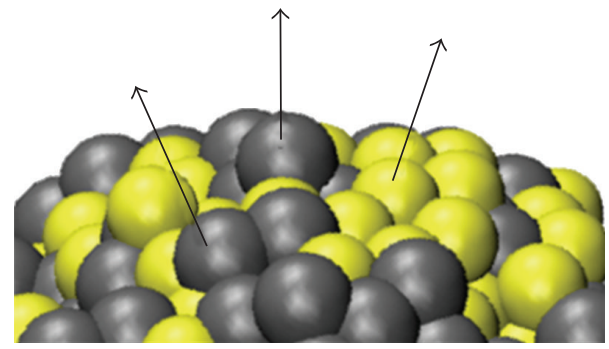

FIGURE 2: Schematic rendering of the surface of an iron-technetium alloy (iron: yellow and technetium: gray) showing schematically that dissolution can involve either technetium or iron atoms and that corrosion processes will involve breaking apart the local bonding arrangements, which will vary from atom to atom.

consulting accurate materials properties for the individual metals and their intermetallic phases, and these can be determined either from experiments, or higher-level theoretical calculations [10]. In the absence of experimental data and materials properties for the intermetallic phase, we have used density functional theory to compute the enthalpy of mixing, bulk modulus, and lattice parameter of a hypothetical B2 phase. We have also performed first-principles calculations of the moduli and lattice parameters of hexagonal closepacked technetium metal. These data-points were then used to construct the rest of the intermetallic iron-technetium modified embedded atom potential, which was then applied to investigate cohesive relations for some model irontechnetium surface systems.

2.1. Density Functional Theory (DFT). Density functional theory is a powerful theoretical technique developed by Hohenberg and Kohn for the determination of the electronic structure associated with a given crystallographic and/or molecular system $[11,12]$. This method is based upon the quantum mechanical physics that describe the interactions occurring between systems of electrons and nuclei. Because the physics behind this method operates on such a fundamental level, the method can very accurately describe the properties of materials that relate to the electronic structure. Such properties include the binding energy that holds a 
material together and the variations in this binding energy that are due to distortions in the structure or composition of a material.

Density functional theory has been implemented in numerous software packages that are commercially available. We utilized the Vienna Ab-initio Simulation Package (VASP) v.4.6.36 to solve for the electronic structures and energies for the reference structures studied in the iron-technetium binary system $[13,14]$. Fully relaxed density functional theory calculations were performed for technetium in the hexagonal close-packed configuration, and materials properties such as the elastic moduli were evaluated by performing small distortions on the unit cell configuration [15]. Similar calculations were performed on a hypothetical iron-technetium (space group B2, Figure 3(b)) intermetallic to provide reference points for the intrinsic properties of iron-technetium metallic systems. Although a sigmaphase intermetallic for the iron-technetium system has been identified by Darby, the low-symmetry disordered structure makes it nontrivial for use in the development of a potential [16]. Furthermore, the materials properties, other than the lattice constant, of the Darby phase have not yet been determined.

Systems parameters within VASP that were specified to expedite the computationally intensive quantum mechanical calculations are described briefly as follows: the projector augmented wave method (PAW) was used to treat the core electrons and core-valence interactions [13]. Planewaves were used as a basis set to expand the electronic structure, with energies taken up to $400 \mathrm{eV}$. The PerdewBurke-Erzenrhof functional (PBE) was used to solve the exchange-correlation contribution to the electronic energy [17]. For the unit cell of hexagonal close-packed technetium, a $13 \times 13 \times 7$ Monkhorst-Pack k-point mesh was used, and for the B2 structure of the hypothetic iron-technetium intermetallic, a $17 \times 17 \times 17 \mathrm{k}$-point mesh was used [18]. Electron smearing was used to accelerate convergence using the Methfessel-Paxton method [19].

2.2. Modified Embedded Atom Method (MEAM). The modified embedded atom method is, in essence, a simplified approach to evaluating the energies and forces on atoms that is achieved by reducing and collecting the equations of density functional theory into a sum of single atom and pairwise expressions [10]. The energy is evaluated by constructing "embedding densities" contributed by all the nearby atoms, based upon simple parameterized forms. Similarly, the pairwise interactions are parameterized by comparison with experimentally or theoretically derived equations of state. The procedure for developing such potentials has been well described in the literature [10]. Because this method is much more computationally expedient than density functional theory, it can be used to simulate materials systems of greater spatial and temporal extent.

A modified embedded atom potential for iron was previously developed by Lee and Baskes [20] and was adopted for this work. Prior to this study, there were no preexisting potentials for technetium and the pairwise irontechnetium interaction. Hence, development of these poten- tials was an important step in this project. The materials properties evaluated using density functional theory were used to determine the necessary modified embedded atom parameters.

The large-scale atomic/molecular massively parallel simulator (LAMMPS), developed at Sandia National Laboratory, was used to perform total energy computations, conjugate gradient optimizations, and molecular dynamics simulations of iron-technetium systems using the modified embedded atom method [21]. To simulate a diversity of irontechnetium surfaces, spherical particles were simulated. Particles of hexagonal close-packed technetium, body-centered cubic iron and B2 iron-technetium were constructed. Technetium particles with iron skins, and iron particles with technetium skins, as well as technetium particles with iron adatoms and iron particles with technetium adatoms were simulated. It was important to consider such a diversity of surface configurations in order to span the entire range of possible local surface configurations that may be relevant to waste form corrosion scenarios. The energy of the surface atoms in these systems was calculated following a general optimization of the atomic coordinates on the basis of a local energy minimization procedure. Furthermore, molecular dynamics simulations at $1000 \mathrm{~K}$ were also performed to anneal the alloyed particles, thereby allowing even more configurations to be sampled.

The modified embedded atom method involves the computation of system energies by summation of individual and pairwise atomic cohesive energies using the formulae

$$
\begin{gathered}
E=\sum_{i} \sum_{j \neq i} \frac{1}{2} \phi\left(r_{i j}\right)+\sum_{i} F\left(\rho_{i}\right), \\
\rho_{i}=\left(\sum_{l=0}^{3} t^{(l)}\left(\rho_{i}^{(l)}\right)^{2}\right)^{1 / 2}, \\
F\left(\rho_{i}\right)=A_{E} E_{c}\left(\frac{\rho_{X}(R)}{\rho_{X}\left(r_{e}\right)}\right) \ln \left(\frac{\rho_{X}(R)}{\rho_{X}\left(r_{e}\right)}\right), \\
\phi(R)=-2 \frac{E_{c}}{z}\left[\left[1+\eta+0.05 \eta^{3}\right] e^{-\eta}\right. \\
\left.+A_{E}\left(\frac{\rho_{i}}{\rho_{X}\left(r_{e}\right)}\right) \ln \left(\frac{\rho_{i}}{\rho_{X}\left(r_{e}\right)}\right)\right], \\
\eta=\alpha\left(\frac{R}{r_{e}}-1\right),
\end{gathered}
$$

with $F\left(\rho_{i}\right)$ being the embedding function of the density at atom $i$ and $\phi\left(r_{i j}\right)$ being the pair function between atoms $i$ and $j$. The density is composed of a sum of $l+1$ spherical harmonic terms, as defined above, and the pairpotential is related to the Rose equation of state, also defined above. Using this formalism, it is, therefore, possible to distinguish between the local energies of surface atoms in different local configurations. Following the simulations described above, the energies of the surface atoms were tabulated and plotted as a function of the number of nearest technetium atoms 


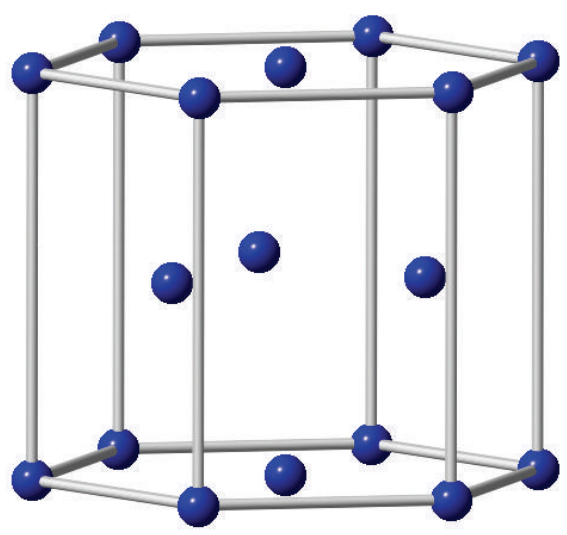

(a)

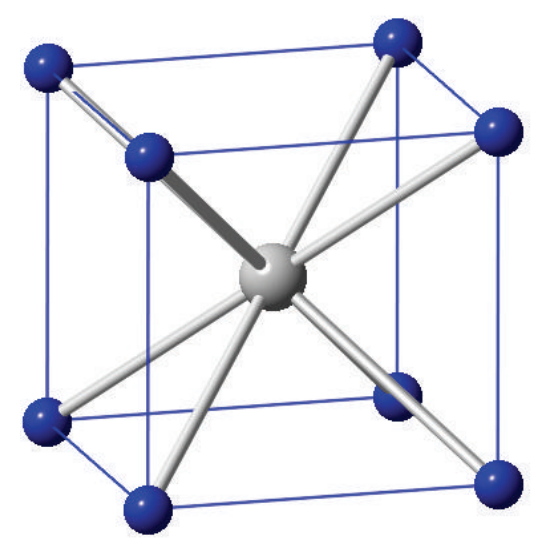

(b)

Figure 3: (a) Hexagonal close-packed crystal structure for Tc, (b) B2 crystal structure for the hypothetical iron-technetium intermetallic used as a reference for development of the modified embedded atom iron-technetium potential.

versus the number of nearest iron atoms. In this way, we were able to discern the physics underlying the relative stabilities of metal atoms in different local orientations.

Attempts to estimate cohesive energies using counts of the number of nearest neighbors have been used in kinetic Monte Carlo simulations of alloy dissolution and nanoporous dealloying [22, 23]. However, in those cases, simple linear relations were assumed to construct the local energy. On the other hand, a modified embedded atom method was combined with a Metropolis Monte Carlo approach by Lillard et al. to simulate crystallographic pitting in hexagonal close-packed metals [24]. By direct computation of the cohesive energies for the alloy systems, in this work, we can directly compare the linear assumptions with the modified embedded atom method energies and determine which atomic configurations and phases will have the greatest impact on the lifetime performance of these alloys.

\section{Results and Discussion}

3.1. Density Functional Theory Computations of Material Properties. Density functional theory computations were performed for the hexagonal close-packed phase of technetium (Figure 3(a)). Following the procedure described by Vitos for hexagonal close-packed materials, the elastic moduli were computed via the finite distortion method [15]. The elastic moduli are reported in Table 1 and compared with the existing experimental values.

Examination of Table 1 indicates that the materials properties calculated using density functional theory for technetium are in reasonable agreement with the experimental results. The moduli are all consistently higher than the experimental values, with the calculated results being closer to the measurements made by Love et al. [25]. We attribute this to the fact that moduli determined from experiment will be sensitive to the temperature at which the experiment is performed. Guillermet reports values determined from experiments performed on Tc metal at room temperature,
TABle 1: Elastic moduli and lattice constants for hexagonal closepacked technetium and selected moduli for the iron-technetium B2 intermetallic taken from density functional theory and experimental data where available.

\begin{tabular}{lcccc}
\hline Tc & DFT & Experiment & Tc-Fe (DFT) & \\
\hline$B, \mathrm{GPa}$ & 320 & $306^{\mathrm{a}}, 281^{\mathrm{b}}$ & $\mathrm{B}, \mathrm{GPa}$ & 290 \\
$E, \mathrm{GPa}$ & 380 & $331^{\mathrm{a}}, 314^{\mathrm{b}}$ & $a, \AA$ & 2.93 \\
$G_{v}, \mathrm{GPa}$ & 146 & $125^{\mathrm{a}}, 123^{\mathrm{b}}$ & $\Delta E^{\mathrm{f}}, \mathrm{eV}$ & +0.325 \\
$V$ & 0.30 & $0.32^{\mathrm{a}}$ & & \\
$c_{11}, \mathrm{GPa}$ & 518 & $433^{\mathrm{b}}$ & & \\
$c_{12}, \mathrm{GPa}$ & 243 & $199^{\mathrm{b}}$ & & \\
$c_{13}, \mathrm{GPa}$ & 193 & $199^{\mathrm{b}}$ & & \\
$c_{33}, \mathrm{GPa}$ & 584 & $470^{\mathrm{b}}$ & & \\
$c_{44}, \mathrm{GPa}$ & 138 & $117^{\mathrm{b}}$ & & \\
$c_{66}, \mathrm{GPa}$ & 131 & & & \\
$a, \AA$ & 2.72 & $2.74^{\mathrm{b}}$ & & \\
$c / a$ & 1.603 & $1.605^{\mathrm{b}}$ & & \\
\hline
\end{tabular}

${ }^{\mathrm{a}}$ Love et al. [25]

${ }^{\mathrm{b}}$ Guillermet and Grimvall [26].

[26] which will be softer than the values by Love et al. obtained at $4 \mathrm{~K}$, which in turn will be softer than the density functional theory values which correspond to the moduli obtained when distorting the lattice about the equilibrium positions at absolute zero.

While the B2 iron-technetium intermetallic is not a known phase for the Tc-Fe binary system, it has composition within the known range of the iron-technetium sigma-phase intermetallic (40\%-60\%), and its symmetry is consistent with the body-centered cubic phase of iron (Figure 3(b)) [16, 27]. Furthermore, the modified embedded atom method has been designed to use this high symmetry phase as a reference for computing the intermetallic terms in the potential energy expression. To develop this potential for the B2 phase, it is not necessary to compute all the elastic moduli (as was the case for technetium). The advantage in this case is due to the 
way that the pairwise intermetallic potentials are derived in the modified embedded atom method: it is only necessary to compute the lattice constant, formation energy, and bulk modulus of the intermetallic phase. We find that the energy of mixing for iron and technetium to produce the B2 phase is $+0.325 \mathrm{eV}$, and that the bulk modulus for this phase is $290 \mathrm{GPa}$, similar to the bulk modulus for hexagonal closepacked technetium metal.

\subsection{Development of the Modified Embedded Atom Potential.} Following the procedure given by Baskes and Johnson [10], we utilize the density functional theory data in Table 1 to develop the modified embedded atom potentials for technetium and the iron-technetium intermetallic and alloy phases. In addition to the moduli and lattice parameters in Table 1, estimates of the vacancy formation energy and cohesive energy were required. The cohesive energy for technetium has been computed from first-principles previously [28]. Since no estimate of the vacancy formation energy of technetium could be found, the vacancy formation energy of rhenium was used instead [10]. Rhenium is commonly used as a surrogate material for investigations of technetium, for reasons of handling and similarity in the materials properties [29]. Vacancy energies can be calculated from first-principles, but they require supercell calculations that are not always practical. In future work, we hope to augment this value with a rigorous vacancy energy calculation. For iron, we utilized the existing potential for body-centered cubic iron created by Lee and Baskes [20]. The resulting MEAM parameters are given in Table 2.

\subsection{Application of MEAM Potential to Map the Surface Sta-} bility of Tc-Fe Alloys. Spherical clusters of technetium, iron, iron-technetium, and various core-shell combinations were modeled using the LAMMPS package. Spherical clusters based on technetium were generated based on a hexagonal close-packed parent lattice. Spherical clusters based on iron were generated using a body-centered cubic lattice, and spherical clusters based on iron-technetium were generated using the hypothetical iron-technetium B2 structure. Geometry optimizations as well as molecular dynamics simulations at $1000 \mathrm{~K}$ for $150 \mathrm{ps}$ were performed on these systems so that the atoms could migrate to low-energy sites. Due to the geometry of the spherical particles, multiple different surface planes (and hence atomic configurations for the surface atoms) were sampled within each simulation. Example clusters are shown in Figure 4.

For the purposes of probing a greater set of surface ensembles, each of the spherical particles shown in Figure 4 was subject to various additional modifications: surface atoms were replaced either completely by technetium, or completely by iron, and randomly selected atoms were switched from one type ( $\mathrm{Tc}, \mathrm{Fe}$ ) to another (Fe, Tc). The cohesive energies calculated for all of the surface atoms within these various ensembles were collated and assembled into a master database. The database consisted of the atom type, the number of nearest neighbors of type Tc, and the number of nearest neighbors of type Fe as well as the cohesive energy for that atom, as determined by the modified
TABLE 2: Parameters for the modified embedded atom potentials for simulation of technetium, iron, and iron-technetium.

\begin{tabular}{lccc}
\hline Parameter & $\mathrm{Tc}$ & $\mathrm{Fe}^{\mathrm{a}}$ & $\mathrm{Tc}-\mathrm{Fe}$ \\
\hline$A_{\text {lat }}$ & 3.853 & 2.866 & 2.537 \\
$E_{0}$ & 7.534 & 4.29 & \\
$A$ & 1 & 0.56 & \\
$\alpha$ & 5.593 & 5.073 & 6.054 \\
$C_{\min }$ & 2.0 & 0.36 & 2.0 \\
$C_{\max }$ & 2.8 & 2.8 & 4.8 \\
$t_{1}$ & 4.209 & 2.6 & \\
$t_{2}$ & 25.8257 & 1.8 & \\
$t_{3}$ & -15 & -7.2 & \\
$b_{0}$ & 1 & 4.15 & \\
$b_{1}$ & 0 & 1 & \\
$b_{2}$ & 0.665 & 1.0008 & +0.325 \\
$b_{3}$ & 3 & 1 & \\
$\Delta E$ & & & \\
\hline
\end{tabular}

${ }^{\mathrm{a}}$ Lee and Baskes [20].

embedded atom potential calculation. For each atom in the simulation, the number of nearest neighbors and their type were determined by counting the number of neighbors with a bond-length less than the sum of the metallic radii for the two atom types (i.e., $r_{\mathrm{Fe}}+r_{\mathrm{Fe}}$, or $r_{\mathrm{Fe}}+r_{\mathrm{Tc}}$, or $r_{\mathrm{Tc}}+r_{\mathrm{Tc}}$ ) plus $10 \%$. Metallic radii were given by half of the nearest neighbor distance in the pure metal (hexagonal close-packed technetium or body-centered cubic iron).

Following this compilation process, we plotted the cohesive energy of technetium and iron atoms that occupy surface sites in the metal versus the number of nearest neighbors of technetium (\#Tc) and the number of nearest neighbors of iron $(\# \mathrm{Fe})$. These cohesive energies are plotted in contour form for the two variables (\#Tc and \#Fe) in Figure 5. Below the cohesive energies I have also plotted the standard deviation in this cohesive energies, based on the variations found in the overall database.

The cohesive energy plots shown in Figure 5 (top) indicate that surface atoms with more nearest neighbors of either iron or technetium possess a lower cohesive energy and, hence, a higher stability as regards to corrosion. This observation is in agreement with recent studies on copper nanoparticles that indicate that an increase in local coordination results in an increase in the cohesive stability of an atom [30]. Areas shown in "hot" colors (reds) correspond to surface environments that will be more likely to corrode. Areas shown in "cool" colors (blues) will be stabilized through stronger local bonding and, therefore, less likely to corrode.

What is also apparent from Figure 5 is that the cohesive energy of a surface atom is not only a function of the number of nearest neighbors, but also on the type of those nearest neighbors. That is, the degree of stabilization granted to an atom by increase coordination will vary depending upon what kind of neighbors the atom is bonding to. For both iron and technetium atoms, surface bonding to technetium provides a much more significant degree of stabilization 


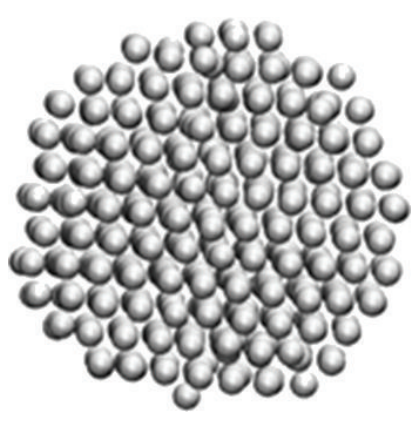

(a)

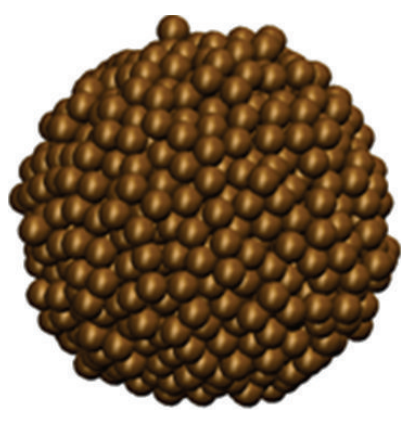

(b)

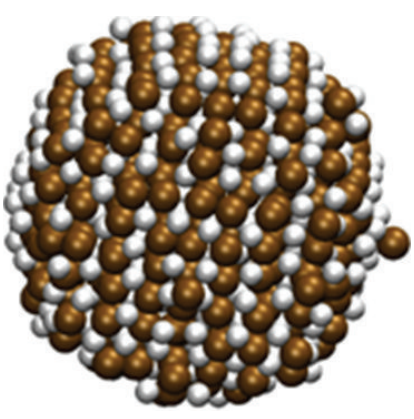

(c)

FIgURE 4: Illustrations of the (a) technetium, (b) iron, and (c) iron-technetium spherical particles used in this work to simulate a variety of surface states. Technetium atoms are rendered in white and iron in brown. The structures shown are the structures obtained following annealing at $1000 \mathrm{~K}$ and conjugate gradient optimization of the atomic positions.

than coordination in an iron-rich surface environment. Thus, technetium-rich surfaces would be expected to be "more noble" than well-mixed or iron-rich surface phases. Recent measurements of iron-technetium candidate alloy waste form corrosion support this observation in that ironrich phases are found to have higher corrosion rates than the intermetallic phases [1].

In the lower portion of Figure 5, we have also plotted the standard deviations in the collated surface atom cohesive energies. This data was plotted to highlight areas in which the assumption that cohesive energies can be determined simply by considering the number and type of nearest neighbors has the potential to break down. For iron surrounded by mostly iron atoms, the standard deviation is relatively small. In this case, an analytical expression for the variation in the cohesive energy as a function of the number of nearest neighbors may still apply. However, as the number of technetium neighbors coordinating the iron atom increases, the standard deviation in the cohesive energy can be as high as $0.5 \mathrm{eV}$. This implies that the orientation, or the ordering, of these nearest neighbors, which is not captured in this two-dimensional representation, is also a significant factor in the stability of those exposed surface atoms. For technetium atoms, the standard deviations are significantly larger than for the iron atoms. This result suggests that the local cohesive energy of technetium atoms is more sensitive to the local configuration of atoms than iron. It may also be an indicator that further testing and development of the modified embedded atom method potential for technetium is required.

\section{Conclusions}

In this work, we have performed density functional theory computations for metallic technetium and iron-technetium intermetallic systems. We have used materials properties obtained from the density functional theory computations to develop a modified embedded atom method potential for the iron-technetium system. The materials properties calculated using density functional theory were in good agreement with the available experimental literature and were, therefore, used to parameterize the MEAM potential.
Simulations containing model surfaces of iron-technetium alloys in the iron-rich, technetium-rich, and 50-50 iron-technetium intermetallic limit were then performed. The simulations were designed to capture the variations in surface cohesive energies that occur as iron or technetium atoms are placed in different local compositional and coordination environments. We collated the local surface atom cohesive energies obtained from the various computations so that we could investigate how the local environments of iron or technetium atoms at the surface of hypothetical irontechnetium alloy waste forms may influence their corrosion behavior. The simulations indicated that the local bonding arrangements are very critical to the ultimate stability of these surface atoms. Generally speaking, increases in local coordination lead to more stable surface atoms; that is, more bonds to nearest neighbors will increase the energy required to dissolve or remove an atom from the alloy surface. Another observation that is revealed by this study is that technetium-rich environments are more noble, not only for technetium atoms on the surface, but also for iron atoms.

Our work also shows that a consideration of the local bonding environment, in terms of numbers of nearest neighbors, is not sufficient for computing or estimating the local cohesive energy and that variations in the particular way the atoms are situated about the central atom can lead to perturbations in the cohesive energy of up to $0.5 \mathrm{eV}$ (as estimated by one standard deviation from the mean). Therefore, although linear relationships for dissolution and diffusion energies have been assumed in previous works, significant errors in these simulations may apply.

The modified embedded atom potential for iron-technetium alloys that we have developed in this work is currently being implemented within a kinetic Monte Carlo scheme, which we will apply in future work for the simulation of relative corrosion rates for iron and technetium in candidate metal alloy waste forms, as well as the differences in corrosion morphology and extending the potential to include other alloy components such as molybdenum and chromium. This technique will allow us to investigate the relative corrosion properties of different intermetallic and alloy phases and the roles of electrochemical potential and $\mathrm{pH}$, and provide a starting point for more complex models 

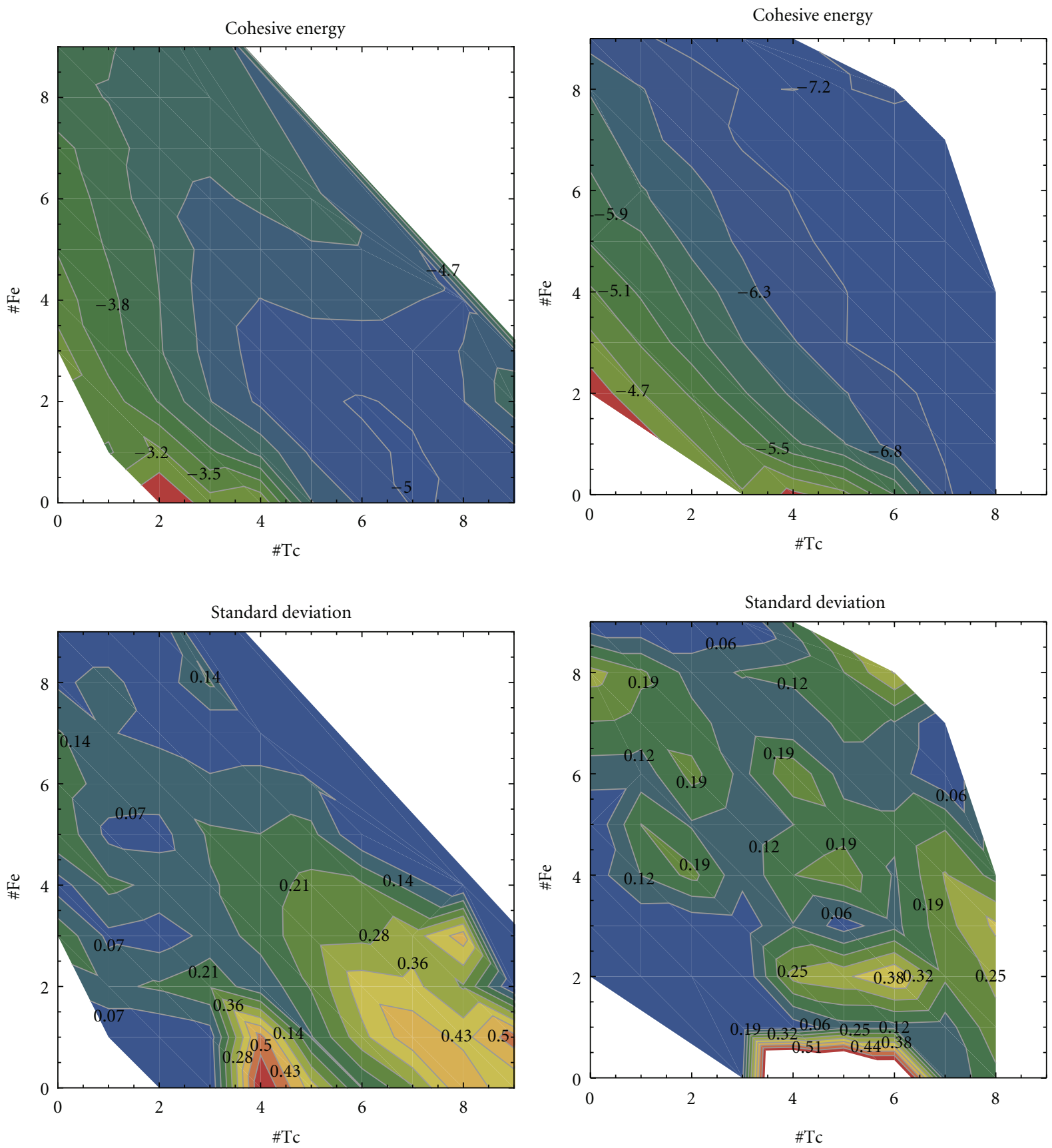

(a)

(b)

FIGURE 5: Cohesive energy contours (upper) and their standard deviation (lower) for iron and technetium components of iron-technetium alloy systems (left and right, respectively) plotted against the number of nearest neighbors of type Tc ( $x$-axis) and Fe ( $y$-axis). Energies and standard deviations are given in $\mathrm{eV}$.

that combine alternative surface processes such as oxidation, grain-boundary dissolution, and surface adsorption.

\section{Acknowledgments}

The authors acknowledge funding through the Fundamental Waste Form Science campaign under the auspices of the US DOE Fuel Cycle R\&D under the direction of John
Vienna, Waste Forms and Separations Campaign Manager. Helpful discussions with Dave Moore, Gordon Jarvinen, Scott Lillard, and Dave Kolman at Los Alamos National Laboratory are also acknowledged. The Los Alamos National Laboratory is operated by Los Alamos National Security LLC for the National Nuclear Security Administration of the U.S. Department of Energy under Contract no. DEAC52-06NA25396. A portion of the research was performed 
using EMSL, a national scientific user facility sponsored by the Department of Energy's Office of Biological and Environmental Research and located at Pacific Northwest National Laboratory.

\section{References}

[1] W. L. Ebert, J. Cunnane, M. Williamson et al., "FY 2010 status report: developing an iron-based alloy waste form," in Fuel Cycle Research and Development, Argonne National Laboratory, Chicago, Ill, USA, 2010.

[2] N. Contributors, "Chart of nuclides," in National Nuclear Data Center, A. A. Sonzogni, Ed., Brookhaven National Laboratory, New York, NY, USA, 2008.

[3] D. Kolman, G. D. Jarvinen, C. D. Taylor et al., "Corrosion and passivity behavior of technetium waste forms," in Corrosion, National Association of Corrosion Engineers, Houston, Tex, USA, 2011.

[4] R. B. Rebak, "Material corrosion issues for nuclear waste disposition in Yucca mountain," Journal of Management, vol. 60, no. 1, pp. 40-43, 2008.

[5] D. Shoesmith, "Fuel corrosion processes under waste disposal conditions," Journal of Nuclear Materials, vol. 282, no. 1, pp. $1-31,2000$.

[6] D. Landolt, "Introduction to surface reactions: electrochemical basis of corrosion," in Corrosion Mechanisms in Theory and Practice, P. Marcus, Ed., pp. 1-18, Marcel Dekker, New York, NY, USA, 2002.

[7] P. F. Weck, E. Kim, F. Poineau, and K. R. Czerwinski, "Structural evolution and properties of subnanometer Tcn ( $n=2-15$ ) clusters," Physical Chemistry Chemical Physics, vol. 11, no. 43, pp. 10003-10008, 2009.

[8] F. Poineau, T. Hartmann, P. F. Weck et al., "Structural studies of technetium-zirconium alloys by X-ray diffraction, highresolution electron microscopy, and first-principles calculations," Inorganic Chemistry, vol. 49, no. 4, pp. 1433-1438, 2010.

[9] C. D. Taylor, "Surface segregation and adsorption effects of iron-technetium alloys from first-principles," Journal of Nuclear Materials, vol. 408, no. 2, pp. 183-187, 2011.

[10] M. I. Baskes and R. A. Johnson, "Modified embedded atom potentials for HCP metals," Modelling and Simulation in Materials Science and Engineering, vol. 2, no. 1, article 011, pp. 147-163, 1994.

[11] W. Kohn and L. J. Sham, "Self-consistent equations including exchange and correlation effects," Physical Review, vol. 140, no. 4, pp. A1133-A1138, 1965.

[12] P. Hohenberg and W. Kohn, "Inhomogeneous electron gas," Physical Review, vol. 136, no. 3, pp. B864-B871, 1964.

[13] G. Kresse and D. Joubert, "From ultrasoft pseudopotentials to the projector augmented-wave method," Physical Review B, vol. 59, no. 3, pp. 1758-1775, 1999.

[14] G. Kresse and J. Hafner, "Ab initio molecular dynamics for liquid metals," Physical Review B, vol. 47, no. 1, pp. 558-561, 1993.

[15] L. Vitos, Computational Quantum Mechanics for Materials Engineers: The EMTO Method and Applications, SpringerVerlag, London, UK, 2007.

[16] J. B. Darby, D. J. Lam, L. J. Norton, and J. W. Downey, "Intermediate phases in binary systems of technetium-99 with several transition elements," Journal of The Less-Common Metals, vol. 4, no. 6, pp. 558-563, 1962.
[17] J. P. Perdew, K. Burke, and M. Ernzerhof, "Perdew, Burke, and Ernzerhof reply," Physical Review Letters, vol. 80, no. 4, pp. 891-891, 1998.

[18] H. J. Monkhorst and J. D. Pack, "Special points for Brillouinzone integrations," Physical Review B, vol. 13, no. 12, pp. 51885192, 1976.

[19] M. Methfessel and A. T. Paxton, "High-precision sampling for Brillouin-zone integration in metals," Physical Review B, vol. 40, no. 6, pp. 3616-3621, 1989.

[20] B.-J. Lee and M. I. Baskes, "Second nearest-neighbor modified embedded-atom-method potential," Physical Review B, vol. 62, no. 13, pp. 8564-8567, 2000.

[21] S. Plimpton, "Fast parallel algorithms for short-range molecular dynamics," Journal of Computational Physics, vol. 117, no. 1, pp. 1-19, 1995.

[22] D. M. Artymowicz, J. Erlebacher, and R. C. Newman, "Relationship between the parting limit for de-alloying and a particular geometric high-density site percolation threshold," Philosophical Magazine, vol. 89, no. 21, pp. 1663-1693, 2009.

[23] J. Erlebacher, "An atomistic description of dealloying porosity evolution, the critical potential, and rate-limiting behavior," Journal of the Electrochemical Society, vol. 151, no. 10, pp. C614-C626, 2004.

[24] R. S. Lillard, G. F. Wang, and M. I. Baskes, "The role of metallic bonding in the cristallographic pitting of magnesium," Journal of the Electrochemical Society, vol. 153, no. 9, pp. B358-B364, 2006.

[25] G. R. Love, C. C. Koch, H. L. Whaley, and Z. R. McNutt, "Elastic moduli and Debye temperature of polycrystalline technetium by ultrasonic velocity measurements," Journal of The Less-Common Metals, vol. 20, no. 1, pp. 73-75, 1970.

[26] A. F. Guillermet and G. Grimvall, "Thermodynamic properties of technetium," Journal of The Less-Common Metals, vol. 147, no. 2, pp. 195-211, 1989.

[27] H. Okamoto, Binary Alloy Phase Diagrams, ASM International, Russell Township, Ohio, USA, 2nd edition, 1990.

[28] V. L. Moruzzi, J. F. Janak, and A. R. Williams, Calculated Electronic Properties of Metals, Pergamon Press, New York, NY, USA, 1978.

[29] J. G. Darab and P. A. Smith, "Chemistry of technetium and rhenium species during low-level radioactive waste vitrification," Chemistry of Materials, vol. 6, no. 5, pp. 1004-1021, 1996.

[30] C. Taylor, M. Neurock, and J. R. Scully, "First-principles investigation of the fundamental corrosion properties of a model $\mathrm{Cu}_{38}$ nanoparticle and the (111), (113) surfaces," Journal of the Electrochemical Society, vol. 155, no. 8, pp. C407C414, 2008. 

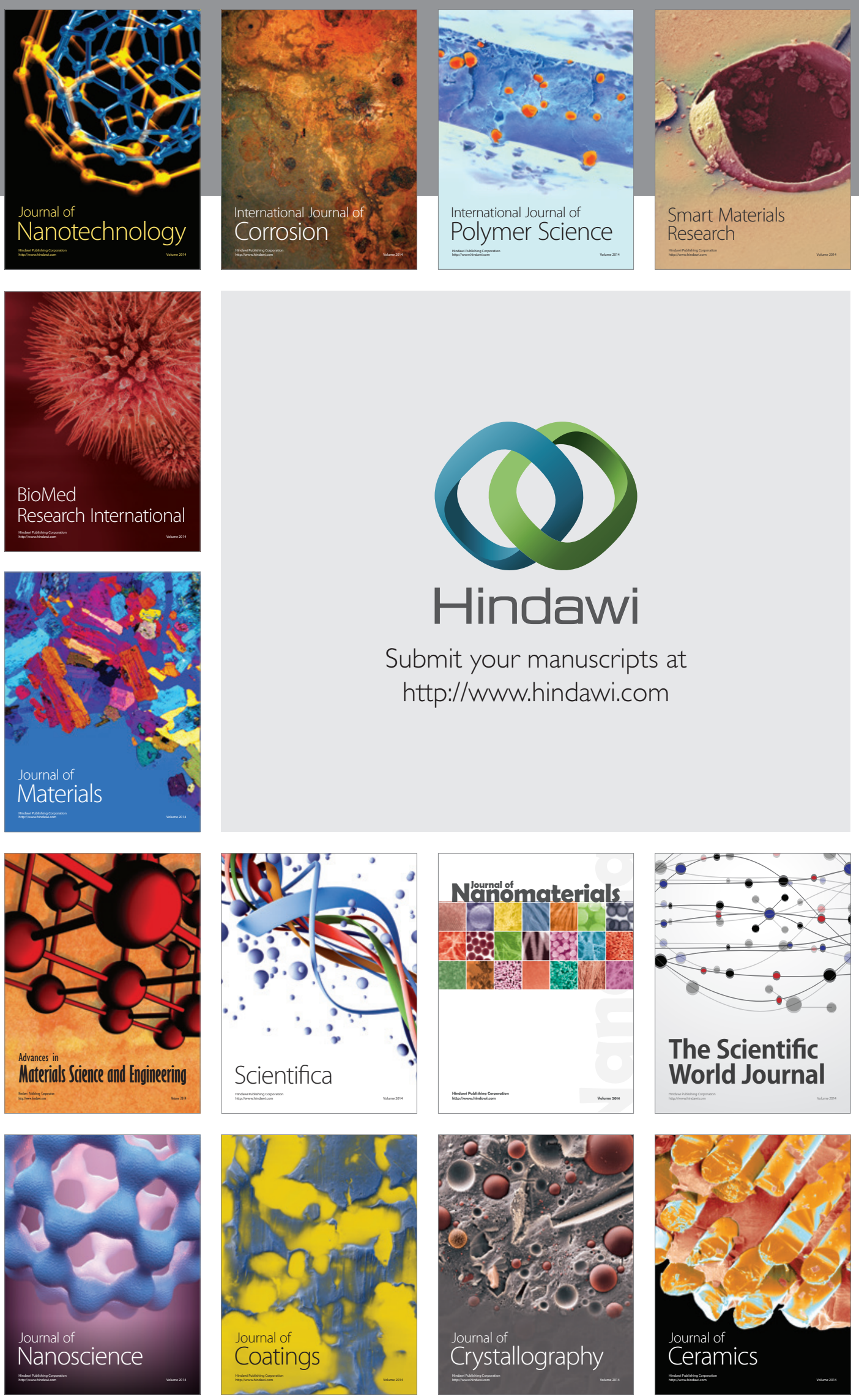

The Scientific World Journal

Submit your manuscripts at

http://www.hindawi.com

\section{World Journal}

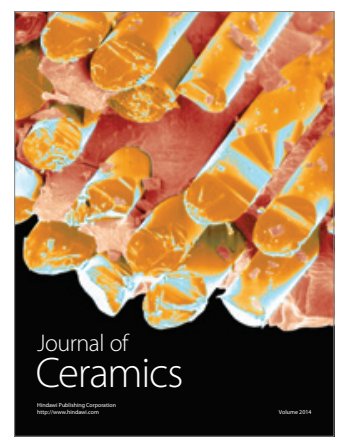

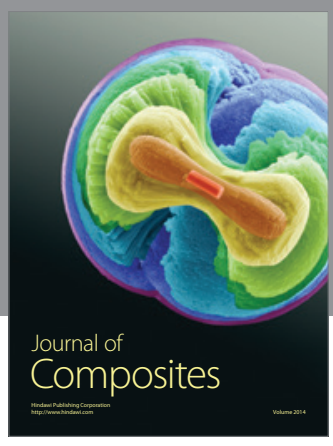
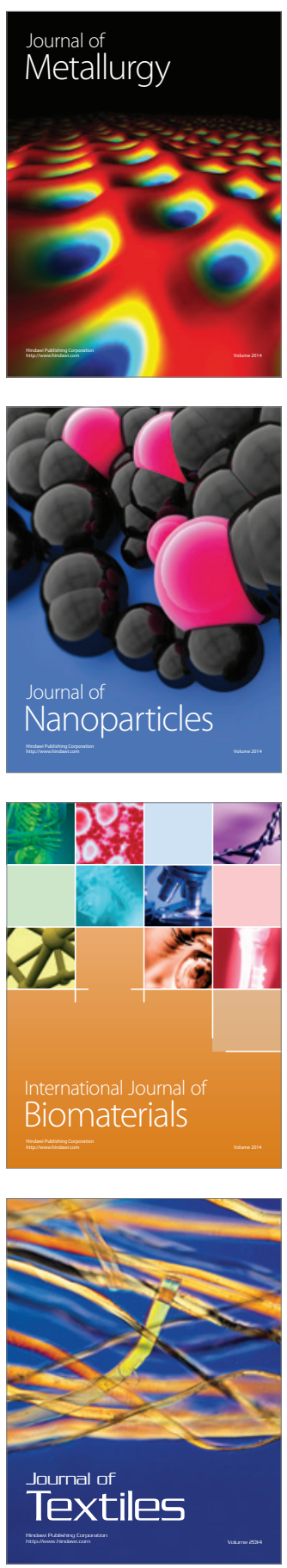